\title{
Diagnostic accuracy of multi-detector row computed tomography and magnetic resonance imaging in assessment of mandibular involvement, cervical lymph node metastases and $T$ parameter staging of squamous cell carcinomas of tongue and floor of mouth: A prospective correlation with postoperative pathologic data
}

\section{Kumar R', Jaiswal $\mathbf{G}^{2}$, Kundu J3}

${ }^{1}$ Ravinder Kumar, Associate Professor, Department of Radiodiagnosis; ${ }^{2}$ Gagan Jaiswal, $3^{\text {rd }}$ Year P.G. Student, Department

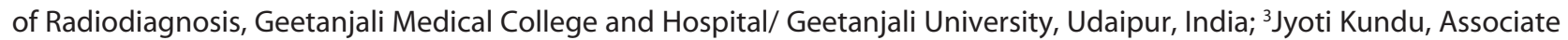
Professor, Department of Prosthodontics, Geetanjali Dental and Research Institute/ Geetanjali University, Udaipur, India.

\begin{abstract}
Background: Tongue and floor malignancies can be diagnosed based on biopsy and clinical examination, but diagnostic imaging is essential to confirm and define the size and extent of the disease.

Objectives: The aim of this study was to recognise and understand the extent and precision of the diagnostic accuracy of multi-detector row computed tomography (MDCT) and magnetic resonance imaging (MRI) in mandibular tumour involvement, cervical lymph node metastases, T staging of tongue and floor of mouth malignancies in order to represent the proper treatment plan.

Methods: Fifty patients with histologically proven squamous cell carcinomas (SCC) of tongue and floor of mouth malignancies underwent both multi-detector row computed tomography and magnetic resonance imaging before surgery. Two radiologists independently, unaware of the findings, in consensus reviewed and interpreted all images to determine the extent of the primary tumour $(\mathrm{T})$, mandibular tumour invasion and cervical lymph node metastasis. Imaging results obtained were correlated with postoperative histopathologic findings that represented our reference standard. Cohen's kappa index and McNemar test were used to verify the concordance between the two radiologists and above mentioned three study parameters.

Results: Magnetic resonance imaging showed superiority to multi-detector row computed tomography in the evaluation of the T parameter staging of TNM classification and medullary bone involvement while multi-detector row computed tomography was more accurate compared to magnetic resonance imaging in the visualization of small cortical bone erosions. However, both the modalities demonstrated similar diagnostic performance in detection of metastatic lymph nodes.

Conclusion: Multi-detector row computed tomography and magnetic resonance imaging have nearly equal potential for detecting the tumour size, mandibular infiltration and nodal metastases.
\end{abstract}

Key words: Lymph nodes, Magnetic Resonance Imaging, Mandibular involvement, Multi-detector row computed tomography, Neoplasm metastasis, Tongue carcinoma

Address for correspondence

Dr. Ravinder Kumar

Associate Professor, Department of Radiodiagnosis

Geetanjali Medical Campus

Manvakhera, Udaipur-313002.

Rajasthan, India.

E-mail: kundu19@yahoo.co.in

\section{INTRODUCTION}

uperficial tongue and floor of mouth lesions can be $\checkmark$ easily accessed and diagnosed by clinical examination and biopsy, because the initial manifestation of most disease is mucosal change. However, characterization and extent of tongue and floor of mouth neoplasms may be recognized only on cross-sectional images such as those obtained by Multi-detector row computed tomography 
(MDCT) or Magnetic resonance imaging (MRI), thus permitting specific radiologic diagnostic work-up for these tumours. Various radiologic techniques, such as sonography, CT, and MR imaging are used to study head and neck tumours, including tongue and floor of mouth neoplasms ${ }^{1-5}$. CT images are degraded by dental amalgams and dense bones ${ }^{3,4}$. Although MR imaging failed in differentiating post-radiation scar tissue from tumour but it has shown superior soft-tissue contrast compared with CT scans $\mathrm{s}^{5-7}$. The presence and extent of nodal metastases, mandibular bone involvement and T-staging in oral and pharyngeal neoplasms has a great impact on treatment and prognosis. Pre-treatment $\mathrm{CT}$ and MRI of the neck are commonly performed to evaluate these parameters in order to optimize and decide treatment planning, therapeutic approach and prognosis of patients. The critical determinant of the utility of an imaging modality for oral cancer is its ability to detect the presence or absence of metastatic lymph nodes, particularly when it is not otherwise clinically evident $^{8}$. Some authors consider MDCT as the firstline examination owing to its high accessibility and reliability ${ }^{9}$ while other authors have mentioned superior diagnostic accuracy of MRI in local staging and detecting metastatic cervical lymph nodes ${ }^{10,11}$.

This article describes the role and precision of MDCT and MRI in the determination of T staging of Squamous cell carcinomas (SCC) of tongue and floor of mouth, using the TNM classification (American Joint Committee Cancer Staging, 1988) ${ }^{12}$ and detecting the diagnostic accuracy of mandibular tumour invasion by prospectively correlating $\mathrm{CT}$ and MRI findings with postoperative histopathologic analysis, that represented our reference standard. It also discusses whether MRI is sub-optimal imaging technique in detecting the metastatic cervical lymph node of SCC of tongue and floor of mouth patients by verifying the concordance of MDCT and MRI evaluation.

\section{METHODS}

Institutional review board approval with a waiver of informed patient consent was obtained.

Fifty patients with histologically proven tongue neoplasm were selected for the study. Forty eight patients underwent glossectomy, and their preoperative CT and MR imaging findings were correlated with postoperative pathologic findings. Special attention was paid to the nodal metastases, mandibular tumour invasion, size and extent of primary tumours. The inclusion criteria for the study were: (i) both surgical procedure and preoperative imaging examinations performed in our institution, (ii) having the results of histopathological examinations and (iii) a clinical evaluation of the mandibular infiltration. Exclusion criteria were: (i) patients who performed only MDCT or only MRI examinations, (ii) lack of histopathological confirmation of tongue tumour, (iii) preoperative treatments with radiotherapy and/ or chemotherapy, (iv) time greater than two weeks between the two examinations and (v) the presence of metallic artefacts in the images that could interfere with radiological interpretation.

MR images were acquired on a 1.5-T (Magnetom, Siemens, Iselin, NJ) superconductive system. MRI was performed using a neck coil, 5-mm (millimetre) thick sections, and a matrix of $256 \times 256$. In the study, spinecho T1-weighted (550/15/2 [repetition time (TR)/echo time (TE)/excitations]) and T2-weighted sequences (2500/15-90/1) were performed in multiple planes (axial and coronal or sagittal) selected in relation to the site of the tumours in the oral cavity and short-tau-inversionrecovery (STIR) sequences T2 weighted (TR 1800ms; TE $100 \mathrm{~ms}$ ) acquired on the axial plane. The protocol also included a spinecho T1-weighted sequence on coronal or axial plane with 3-mm thick slices for the evaluation of the mandible. In addition, for the study of tumour extension to the mandible, gradient-echo sequences $\left(190 / 20,25^{\circ}\right.$ flip angle) in the axial planes were used.

MDCT examination was performed using a 64-MDCT scanner (Somatom sensation 64, Siemens Healthcare, Germany). The scans were performed with the patient supine and neck hyper-extended. The parameters used were: slice collimation $4 \times 1$; tube voltage 120-140 kV; effective mAs 150; slice thickness $1 \mathrm{~mm}$. Reconstructed slices at $1.5-\mathrm{mm}$ intervals; gantry rotation time $0.8 \mathrm{~s}$; were secondarily obtained with a field of view of $35 \mathrm{~cm}$ focused on the oral cavity structures.

MDCT diagnostic criteria used for the evaluation of the mandibular bone invasion were: (i) demonstration of cortical bone defects adjacent to the tumour, in order to determine the cortical invasion, (ii) evidence of trabecular disruption continuous to the cortical bone erosion, in order to determinate the marrow involvement and (iii) MDCT infiltration signs of the inferior alveolar canal.

MRI diagnostic criteria used for the evaluation of infiltration of mandibular bone were: (i) the lack of typical hypointense signal of cortical bone on $\mathrm{T} 1$ and $\mathrm{T} 2$ replaced by the signal intensity of the tumour, useful to determinate the cortical invasion, (ii) the presence of MRI infiltration signs of the inferior alveolar canal. 
MDCT and MRI criteria for the diagnosis of metastatic lymph node ${ }^{13,14}$ : (i) any lymph node greater than 10 $\mathrm{mm}$ in maximum diameter, (ii) presence of central nodal necrosis, ( iii) indistinct nodal margins and (iv) loss of fatty hilum.

T stages were determined in all patients. For the staging of tumours of the tongue, the American Joint Committee Cancer Staging criteria for oral cavity tumours were used. The criteria for staging oropharynx tumours were used for the evaluation of tumours of the base of the tongue and floor of mouth.

This was a prospective study; the results, obtained with MDCT and MR, were compared by two radiologists independently, unaware of the histopathological findings, in consensus reviewed and interpreted all images to determine the results, which were then successively correlated with pathologic data.

\section{STATISTICAL ANALYSIS}

The results of radiological assessment were correlated with histopathologic results by Chi-square test. Sensitivity, specificity, positive predictive value (PPV) and negative predictive value (NPV) of MRI and MDCT were assessed for mandibular tumour invasion. For qualitative analysis, McNemar test was used to evaluate the overall accuracy of both imaging techniques in the evaluation of the study parameters. Inter-observer agreement as regards $T$ staging, presence of metastatic cervical lymph node involvement and mandibular tumour infiltration was analysed using Cohen's kappa index. A statistical significance level of $95 \%$ ( $p$ value $<0.05$ ) and a $5 \%$ error level were considered for the analyses. Interpretation criteria of the kappa index recommended by Landis and Koch ${ }^{15}$ were used to analyse the results. Differences in the accuracy, sensitivity, specificity, PPV and NPV were calculated at a statistical significance of $p<.05$.

Statistical analysis was performed with the Statistical Package for Social Sciences 13.0 (SPSS, Chicago, IL, USA).

\section{RESULTS}

Fifty patients (36 men, 14 women; mean age, 57 years; range, $25-75$ years) with histologically proven tongue neoplasm, who performed both a preoperative MRI and MDCT, composed our study population (Table 1). Forty eight patients underwent glossectomy, and their preoperative $\mathrm{CT}$ and MR imaging findings were correlated with postoperative pathologic findings. The clinical stage is indicated for two patients who did not undergo surgery.

\section{MANDIBULAR INVASION}

Pathological examination showed evidence of mandibular invasion in $18(38 \%)$ patients while no bone involvement was demonstrated in 30 (62\%) patients. Table 2 showed the sensitivity, specificity, accuracy, PPV and NPV of MDCT and MRI in the assessment of mandibular invasion.

MRI exhibited better results than MDCT although no statistically significant difference was noted ( $p>.05$ ). Also, McNemar test and kappa index (Table 4) did not show any difference in the diagnostic accuracy for the evaluation of mandibular invasion between the two modalities ( $p>.05)$.

\section{Table 1: Demographic, clinical and pathological findings of patients from the study}

\begin{tabular}{|c|c|c|c|}
\hline \multicolumn{3}{|c|}{ Age (years); Mean (range) } & $57 ;(25-75)$ \\
\hline \multicolumn{4}{|l|}{ Gender (n) } \\
\hline \multicolumn{3}{|l|}{ Male } & 36 \\
\hline \multicolumn{3}{|l|}{ Female } & 14 \\
\hline \multicolumn{3}{|c|}{ Weight ( kg); Mean (range) } & $62 ;(47-75)$ \\
\hline \multicolumn{3}{|c|}{ Body mass index $\left(\mathrm{kg} / \mathrm{m}^{2}\right)$; Mean (range) } & $23 ;(18-28)$ \\
\hline \multicolumn{3}{|c|}{ Time interval between MDCT \& MRI examinations (days) } & $8 ;(2-14)$ \\
\hline \multicolumn{4}{|c|}{ T stage of tumours of the tongue and floor of the mouth $(n=50)$ evaluated clinically ( 2 cases) and pathologically ( 48 cases). } \\
\hline T Stage & Tongue & Base of the tongue & Floor of the mouth \\
\hline T1 & 7 ( 6 pathological, 1 clinical case ) & 1 (1 clinical case ) & 1 \\
\hline $\mathrm{T} 2$ & 6 & 5 & 2 \\
\hline T3 & 2 & 7 & 1 \\
\hline $\mathrm{T} 4$ & 0 & 6 & 12 \\
\hline Total & 15 & 19 & 16 \\
\hline
\end{tabular}


Table 2: Sensitivity, specificity, accuracy, positive predictive value and negative predictive value of multi-detector row computed tomography and magnetic resonance imaging in the assessment of mandibular invasion.

\begin{tabular}{|ccc}
\hline & Multi-detector row computed tomography & Magnetic resonance imaging \\
\hline Sensitivity & $83.3 \%[15 / 18]$ & $88.8 \%[16 / 18]$ \\
\hline Specificity & $90.0 \%[27 / 30]$ & $93.3 \%[28 / 30]$ \\
\hline Accuracy & $87.5 \%[42 / 48]$ & $91.6 \%[44 / 48]$ \\
\hline Positive predictive value & $83.3 \%[15 / 18]$ & $88.8 \%[16 / 18]$ \\
\hline Negative predictive value & $90.0 \%[27 / 30]$ & $93.3 \%[28 / 30]$ \\
\hline
\end{tabular}

Table 3: Comparison of multi-detector row computed tomography and magnetic resonance imaging with pathologic T stage

\begin{tabular}{|c|c|c|c|c|c|c|c|}
\hline \multirow[t]{2}{*}{ MR T Stage } & \multirow[t]{2}{*}{$\mathbf{N}$} & \multicolumn{6}{|c|}{ Pathologic T Stage (48 cases) } \\
\hline & & $\mathrm{T} 1$ & $\mathrm{~T} 2$ & T3 & & T4 & \\
\hline $\mathrm{T} 1$ & 6 & 5 & 1 & - & & - & \\
\hline $\mathrm{T} 2$ & 14 & 2 & 12 & - & & - & \\
\hline T3 & 10 & - & - & 8 & & 2 & \\
\hline $\mathrm{T} 4$ & 18 & - & - & 2 & & 16 & \\
\hline \multirow[t]{2}{*}{ MDCT T Stage } & $\mathbf{n}$ & \multicolumn{6}{|c|}{ Pathologic T Stage (48 cases) } \\
\hline & & TO & T1 & $\mathrm{T} 2$ & T3 & & $\mathrm{T} 4$ \\
\hline $\mathrm{T} 1$ & 6 & - & 4 & 2 & - & & - \\
\hline $\mathrm{T} 2$ & 14 & - & 3 & 11 & - & & - \\
\hline T3 & 10 & - & - & - & 7 & & 3 \\
\hline $\mathrm{T} 4$ & 18 & - & - & - & 3 & & 15 \\
\hline
\end{tabular}

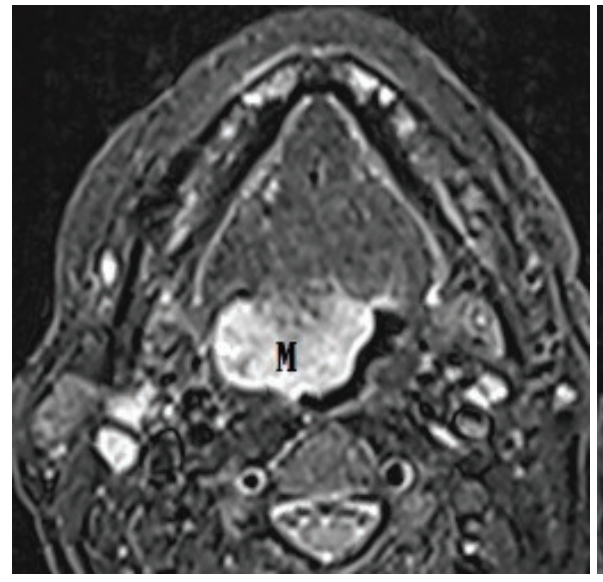

Figure 1(a)

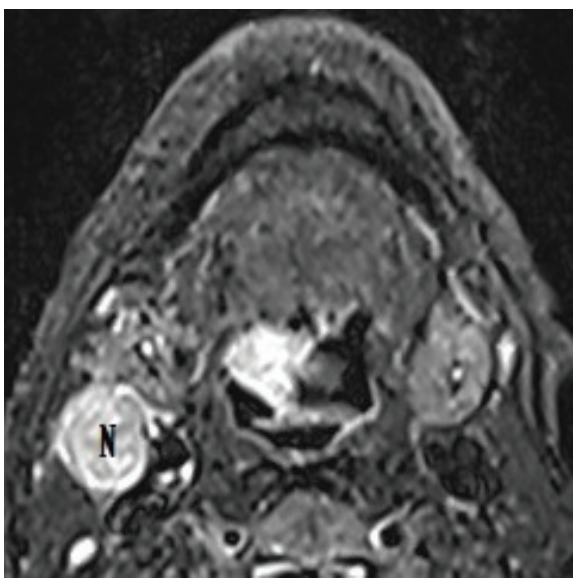

Figure 1(b)

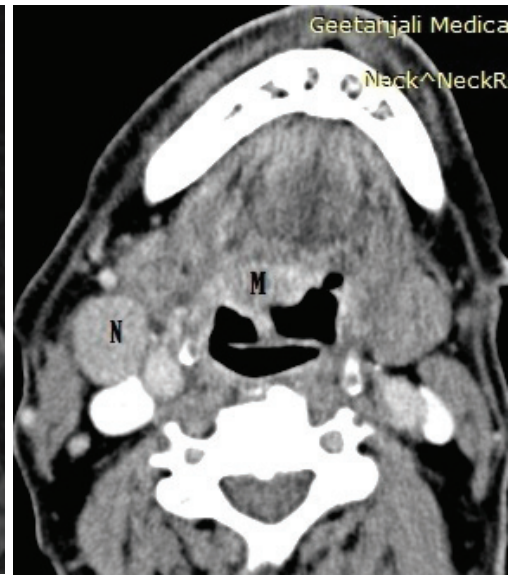

Figure 1(c)

Figure 1: SCC base of tongue (Path: T2) was staged correctly by both CT (T2) and MR (T2) staging, MR is superior to CT in tumoural border delineation and metastatic lymph node detection.

Figure 1 (a \& b): Axial STIR imageshowed high signal intensity tumoural lesion over base of tongue (M) and enlarged right submandibular lymph node $(\mathrm{N})$.

Figure 1(c): Axial post contrast CT scan shows enhancing metastatic lymph node and mass.Both CT\& MR suggested T2N1 stage. 


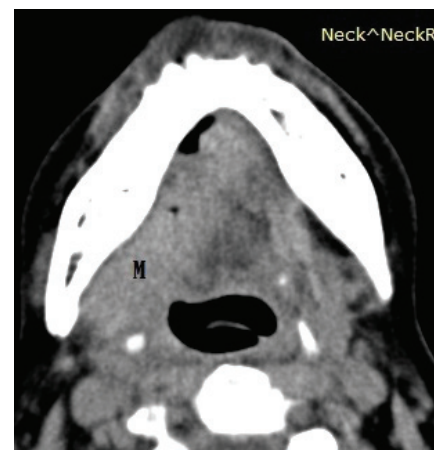

Figure 2(a)

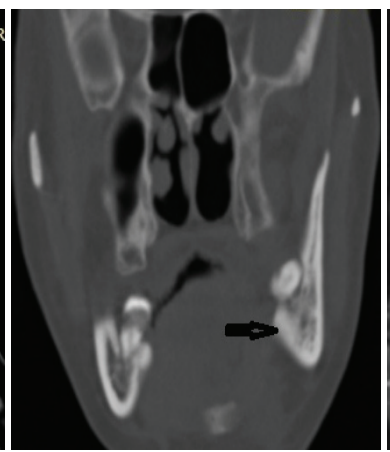

Figure 2(b)

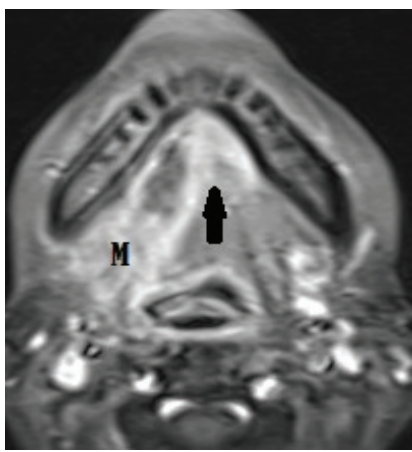

Figure 2(c)

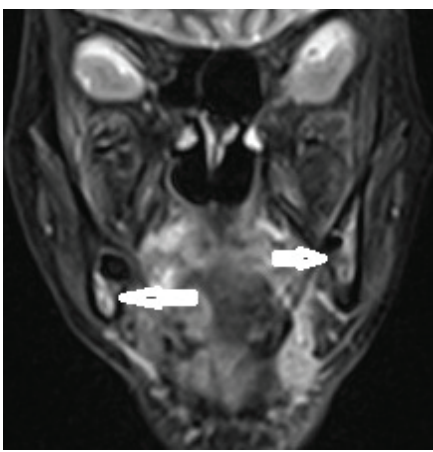

Figure 2(d)

Figure 2: Tumour of the floor of mouth: Stage T4 by pathology.

Figure 2(a): Axial contrast CT scan showed anenhancing lesion over right tongue border and floor of mouth (M).

Figure 2(b): Coronal CT section clearly suggeststumour invading the mandible with cortical erosion and medullary bone involvement (arrow).

Figure 2(c): Axial contrast enhanced MR image shows enhancing mass in right sided floor of mouth (M).It reveals clear demarcation of tumour crossing the midline anteriorly (blackarrow) while Figure 2(d): Coronal STIR section shows marrow oedema in the mandible involving both rami, suggestive of mandibular infiltration by the tumour. This patient underwent subtotal glossectomy and the findings on MR agreed with pathologic findings.

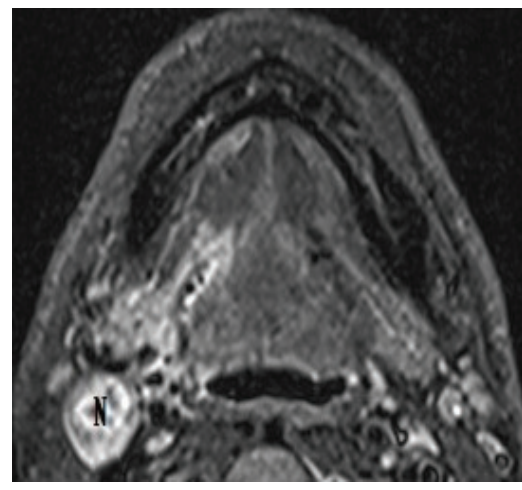

Figure 3(a)

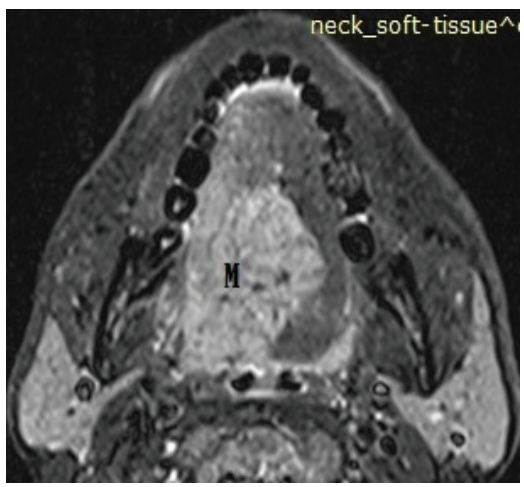

Figure 3(b)

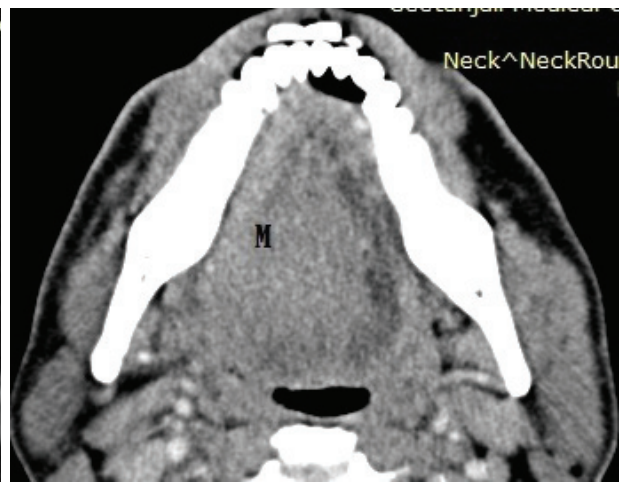

Figure 3(c)

Figure 3: Tumour of the tongue: Stage T4 by pathology.

Figure 3(a): Axial STIR MR image shows well defined, enlarged right submandibular lymph node with sign of colliquation (N). Also, seen is extensivetumoural lesion over right border of tongue.

Figure 3(b): Axial contrast enhanced T1WI MR image revealsenhancing mass in right sided tongue (M), widely extending towards the base of tongue and retromolar trigone of mandible without crossing the midline.

Figure 3(c): Axial contrast CT scan showed an enhancing lesion over right tongue border and base of tongue (M) with no sign of adjacent bony cortical destruction

\section{T-PARAMETER STAGING OF TNM CLASSIFICATION}

MDCT and MR allowed good correlation of the T-staging with pathologic data (48 cases; as 2 clinical cases, not undergoing surgery, were excluded). See fig. 1 and 2.

In 11 of 48 patients, MDCT did not correlate well with pathologic $T$ stages and MR did not allow accurate prediction of the stages in 7 of 48 cases. Six cases were over staged by MDCT. In three out of six cases, mandibular invasion (T4) was suspected by MDCT studies, but no definite evidence of bony invasion was detected by pathology ( $n=3, C T: T 4$, Path: T3) while in the other three cases, $1.8 \mathrm{~cm}, 1.5 \mathrm{~cm}$ and $1.6 \mathrm{~cm}$ superficial lesions of tongue were oversized as $2.2 \mathrm{~cm}, 2.0 \mathrm{~cm}$ and $2.1 \mathrm{~cm}$ respectively $(\mathrm{n}=3, \mathrm{CT}: \mathrm{T} 2$, Path:T1). In three cases, 
MDCT analysis did not demonstrate the infiltration of the marrow by alveolar ridge without a cortical erosion $\{\mathrm{n}=3, \mathrm{CT}: \mathrm{T} 3$, Path: T4. See fig 3 (c)\}; in three cases $(\mathrm{n}=3)$, small superficial lesions of the tongue $(2.3 \mathrm{~cm}[\mathrm{CT}: \mathrm{T} 1$, Path: T2] and $2.5 \mathrm{~cm}$ [ $\mathrm{CT}: \mathrm{T} 1$, Path: T2] ) were not reported and this resulted in five false negative cases at MDCT. On MRI imaging, 2 cases were not confirmed at histopathological examination and they resulted in 2 false positives, either because of the supposed marrow infiltration ( $n=1, M R: T 4$, Path: T3) or the supposed cortical erosion ( $n=1, M R: T 4$, Path: T3). In two lesions, MR studies interpreted stage T2 whereas pathologically it was found to be T1 $(n=2)$. At MRI, there was one false negative $(n=1)$ because superficial lesion of tongue [2.1 cm (MR: T1, Path: T2)] was not visualised on MR while in two cases bony invasion was confirmed pathologically but MR demonstrated no bony invasion $\{n=2, M R$ : T3; Path: T4. See figure 3(a\&b)\} and Table 3.

\section{CERVICAL LYMPH NODE METASTASES}

In detection of number of lymph node metastases, no statistically significant difference was calculated between the accuracy of MDCT and MR using the Cohen's kappa index and McNemar test $(k=.862)$. Furthermore, diagnostic accuracy of MDCT and MRI was equal in detecting the number of metastatic lymph nodes $(n=33$, size $>10 \mathrm{~mm}$ ). There is no significant concordance between CT scan and MRI in estimation of metastatic lymph node size. However, on pathological examination, 3 out of 33 lymph nodes proved to be reactive and not metastatic. The mean size of lymph nodes in MDCT is $15.64 \mathrm{~mm}$ and in MRI scan, it is 15.92 $\mathrm{mm}$.

\section{DISCUSSION}

In the pertinent light of literature, only few studies controversially discussed the focus, significance, value and domain of MDCT and MR in pre-operative tumour staging of oral SCC. The results of the present study contribute to recognizing the diagnostic importance of MDCT and MR examination in establishing the staging of tongue cancer, bone involvement detection and interpretation of metastatic lymph nodes. Tumours of the oral cavity and oropharynx are very aggressive, clinically characterized by diverse morphological features and pathologically characterized with marked local invasiveness, producing metastases in the cervical region ${ }^{16}$.

An accurate staging work-up for the planning of surgical or treatment management, in tumours of the tongue and floor of mouth, comes when the clinical mucosal extent, based on visual and tactile observations, is matched with the radiographic evaluation of deeptissue extent. Despite the fact that biopsy and clinical examination achieves a definitive diagnosis of tongue cancer, it cannot elucidate bone invasion, so a preoperative diagnosis of mandibular involvement relies on imaging. MR, MDCT, PET/CT, orthopanthomography, or $\mathrm{CBCT}$ is usually required for confirmation ${ }^{17}$. Any missed diagnosis could cause devastating outcomes but misdiagnosis can adversely affect the prognostic information.Treatment planning is altered by the extent of the tumour across the midline of the lingual septum and to the adjacent structures. Many surgeons recommend a partial glossectomy rather than a total glossectomy for a satisfactory quality of life for patients with tongue cancer. Therefore, it is clinically important to determine the extent and spread of tongue cancer. MR delineates the midline as a linear high intensity structure and the relationship between the tumour and the midline is recognized without difficulty ${ }^{5,12}$. In contrast, the midline is not easily visualized and correctly assessed by MDCT.

However, when the tumour extends and infiltrates to the adjacent structures, treatment planning is altered. Invasion to the mandible requires either marginal mandibulectomy (entails resecting the cortical plate of the mandible adjacent to the tumour, if the tumour is fixed to cortical plate) or segmental mandibulectomy. When the tumour infiltrates to the larynx, the surgeon may perform laryngectomy and/ or partial pharyngectomy. T2-weighted STIR images clearly delineate the extension of tumour into the adjacent extrinsic tongue muscles, pharyngeal constrictor muscles and faucial pillars. T1weighted MR images are useful for assessing tumour extension to the adjacent tissues of high fat content, such as the soft palate, pre-epiglottic space, tonsillar bed, valleculae, and parapharyngeal space. Tumour infiltration to the mandible is demonstrated as the loss of normal low signal intensity from the cortical bone ${ }^{5}$. MDCT scans with Denta scan may offer an excellent technique for the evaluation of bone erosion from tumour $^{19}$. However, studies reported variations in the diagnostic accuracy of MDCT and MRI in detecting mandibular invasion7, 20, 21. A previous study on the evaluation of the tumours of oral cavity by Wiener et al. ${ }^{22}$ suggested MRI superior to MDCT either in the accuracy or in sensitivity while MDCT showed similar specificity compare to MRI. Our study results are not completely in accordance with other researches. Instead, we are more in agreement with the study of Bolzoni et al. where MRI has a higher sensitivity, accuracy and specificity (93\%); 
the predictive positive value was $87.5 \%$ and the negative value was $96 \%{ }^{23}$. Furthermore, our study results are not influenced by dental amalgam or other chemical shift artefacts that could mimic a mandibular tumour invasion and hence supposed to be more accurate.

Our study comprises several potential limitations that merit considerations. First, the methodological limitations inherent the results to be confirmed with larger sample size prospective studies. Second, our examinations were conducted with conventional MRI image and we are in accordance with Bolzoni et al. that high-resolution images might show better details of the mandible and improve the diagnostic accuracy of MR imaging ${ }^{23}$. However, several studies reported a potential limitation of spatial resolution between MDCT and MRI. In diagnosis of oral cavity region, $M R$ imaging with high-resolution $M R I$ and small-diameter surface coils is still shown to have lower spatial resolution than 1-mm-thick CT. Furthermore, the image analysis in our study was qualitative but a quantitative assessment must be made about the depth and grade of the possible infiltration of the mandible. Finally, in our study, we reported 2 false positive cases at MRI in the evaluation of mandibular involvement. The presumable explanations of these readings were attributed to inflammatory changes due to odontogenic disease such as dental caries, severe periodontal disease or tooth extraction. The inflammatory changes create hypointense signal intensity on $\mathrm{T} 1$, hyperintense in T2 sequences and contrast enhancement. This aspect of hypointensity on T1 and contrast enhancement is similar to neoplastic infiltration. The above explanation also justified the detection and interpretation of 3 cases of false positive cervical metastatic lymph nodes. In this study, MRI potential for diagnosis of cervical lymph node metastasis is equal to MDCT; the reason being diagnostic criteria used in the interpretation of CT and MR for lymph nodal metastases, which include any node greater than $10 \mathrm{~mm}$ in maximum diameter, presence of central necrosis, indistinct nodal margins and loss of fatty hilum ${ }^{13,14}$. Various authors suggested different size criteria for staging lymph nodes with $C T$ and MR imaging ${ }^{24,25}$. However, it is often difficult to distinguish reactive lymph node from metastatic ones using these criteria. We believe that further investigations with high resolution MRI and larger study population should be performed.

\section{CONCLUSION}

To summarize, the current study suggests that MRI could offer additional value in the evaluation of the T parameter staging of TNM classification and medullary bone involvement while MDCT is more accurate compared to MRI in the visualization of small cortical bone erosions, though both the modalities demonstrated similar diagnostic performance in detection of metastatic lymph nodes. However, we believe that further investigations with high resolution MRI and larger study population should be performed because of the importance of correct SCC staging at imaging for proper treatment plan.

\section{REFERENCES}

1. Mettler FA, Schultz K, Kelsey CA, Khan K, Sala J, Kligerman M. Grayscale ultrasonography in the evaluation of neoplastic invasion of the base of the tongue. Radiology.1979; 133:781-4.

2. Bruneton JN, Raux P, Caramella E, Manzino JJ, Valicioni J, Demard F. Tongue and tonsil cancer: staging with US. Radiology.1986; 158:743-6.

3. Larsson SG, Mancuso A, Hanafee W. Computed tomography of the tongue and floor of mouth. Radiology.1982; 143:493-500.

4. Muraki AS, Mancuso AA, Harnsberger HR, Johnson LP, Meads GB. CT of the oropharynx, tongue base, and floor of mouth. Radiology.1983; 148:725-31.

5. Lufkin RB, Wortham DG, Dietrich RB, Hoover LA, Larrsen SG, Kangarloo $H$, et al. Tongue and oropharynx: Findings on MR imaging. Radiology. 1986; 161:69-75.

6. Stark DO, Moss AA, Gamsu G, Clark OH, Gooning GW, Webb WR. Magnetic resonance imaging of the neck: Part I. Normal anatomy. Radiology. 1983; 150:447-54.

7. Goerres GW, Schmid DT, Schuknecht B, Eyrich GK. Bone invasion in patients with oral cavity cancer: Comparison of conventional CT with PET/CT and SPCET/CT. Radiology. 2006; 237:281-7.

8. Roh JL, Yeo NK, Kim JS, Lee JH, Cho KJ, Choi SH, et al. Utility of 2-[18F] fluoro-2-deoxy-D-glucose positron emission tomography and positron emission tomography/computed tomography imaging in the preoperative staging of head and neck squamous cell carcinoma. Oral Oncol 2007; 43:887-93.

9. Monnet $\mathrm{O}$, Cohen F, Lecorrolleri T, Vidal V, Jacquier A, Gaubert JY, et al. Cervical lymph nodes. J Radiol 2008; 89:1020-36. 
10. Vogl T, Bisdas S. Lymph node staging. Top Magn Reson Imaging 2007; 18:303-16.

11. King AD, Ahuja AT, Yeung DK, Fong DK, Lee YY, Lei $\mathrm{Kl}$, et al. Malignant cervical lymphadenopathy: diagnostic accuracy of diffusion-weighted MR imaging. Radiology 2007; 245:806-13.

12. NCCN Guidelines Version 1. Cancer of the Oral Cavity, National Comprehensive Cancer Care Clinical Practice Guidelines in Oncology (NCCN Guidelines) 2012 NCCN.org.

13. Curtin HD, Ishwaran H, Mancuso AA, Dalley RW, Caudry DJ, McNeil BJ. Comparison of CT and MR imaging in staging of neck metastases. Radiology. 1998; 207(1): 123-30.

14. Som PM. Detection of metastasis in cervical lymph nodes: $C T$ and MR criteria and differential diagnosis. AJR Am J Roentgenol. 1992; 158:961-9.

15. Landis JR, Koch GG. The measurement of observer agreement for categorical data. Biometrics. 1977; 33(1):159-74.

16. Shin $M$, Matsuo $K$, Tada $T$, Fukushima $H$, Furuta $\mathrm{H}$, Ozeki $\mathrm{S}$, et al. The inhibition of RANKL/RANK signaling by osteoprotegerin suppresses bone invasion by oral squamous cell carcinoma cells. Carcinogenesis.2011; 32(11): 1634-40.

17. Huang SH, Chien CY, Lin WC, Fang FM, Wang PW, Lui CC, et al. A comparative study of fused FDG PET/ MRI, PET/CT, MRI, and CT imaging for assessing surrounding tissue invasion of advanced buccal squamous cell carcinoma. Clin Nucl Med. 2011; 36(7): 518-25.
18. Unger JM. The oral cavity and tongue: magnetic resonance imaging. Radiology 1985; 155:151-53.

19. Brockenbrough JM, Petruzzelli GJ, Lomasney L. DentaScan as an accurate method of predicting mandibular invasion in patients with squamous cell carcinoma of the oral cavity. Arch Otolaryngol Head Neck Surg. 2003; 129:113-7.

20. Lenz M, Greess H, Baum U, Dobritz M, KerstingSommerhoff B. Oropharynx, oral cavity, floor of the mouth: CT and MRI. Eur J Radiol. 2000; 33:203-15.

21. Imaizumi A, Yoshito N, Yamada I. A potential pitfall of MR Imaging for assessing mandibular invasion of squamous cell carcinoma in the oral cavity. AJNR Am J Neuroradiol. 2006; 27:114-22.

22. Wiener $E$, Pautke C, Link TM, Neff A, Kolk A. Comparison of 16-slice MSCT and MRI in the assessment of squamous cell carcinoma of the oral cavity. Eur J Radiol. 2006; 58:113-8.

23. Bolzoni A, Cappiello J, Piazza C. Diagnostic accuracy of magnetic resonance imaging in the assessment of mandibular involvement in oral-oropharyngeal squamous cell carcinoma. Arch Otolaryngol Head Neck Surg. 2004; 130:837-43.

24. Steinkamp H J, Hosten N, Richter C, Schedel H and Felix R.Enlarged cervical lymph nodes at helical CT. Radiology. 1994; 191(3):795-8.

25. Snow GB, Patel P, Leemans CR, Tiwari R.Management of cervical lymph nodes in patients with head and neck cancer. Eur Arch Otorhinolaryngol. 1992; 249(4):187-94.. 